\title{
Comparison of deep learning with regression analysis in creating predictive models for SARS-CoV-2 outcomes
}

\author{
Ahmed Abdulaal ${ }^{1 \dagger}$, Aatish Patel $^{1 \dagger}$, Esmita Charani ${ }^{2}$, Sarah Denny ${ }^{1}$, Saleh A. Alqahtani ${ }^{3,4}$, Gary W. Davies ${ }^{1}$,
} Nabeela Mughal ${ }^{1,2,5}$ and Luke S. P. Moore ${ }^{1,2,5^{*}}$ (D)

\begin{abstract}
Background: Accurately predicting patient outcomes in Severe acute respiratory syndrome coronavirus 2 (SARSCoV-2) could aid patient management and allocation of healthcare resources. There are a variety of methods which can be used to develop prognostic models, ranging from logistic regression and survival analysis to more complex machine learning algorithms and deep learning. Despite several models having been created for SARS-CoV-2, most of these have been found to be highly susceptible to bias. We aimed to develop and compare two separate predictive models for death during admission with SARS-CoV-2.
\end{abstract}

Method: Between March 1 and April 24, 2020, 398 patients were identified with laboratory confirmed SARS-CoV-2 in a London teaching hospital. Data from electronic health records were extracted and used to create two predictive models using: (1) a Cox regression model and (2) an artificial neural network (ANN). Model performance profiles were assessed by validation, discrimination, and calibration.

Results: Both the Cox regression and ANN models achieved high accuracy (83.8\%, 95\% confidence interval (CI) 73.8-91.1 and 90.0\%, 95\% Cl 81.2-95.6, respectively). The area under the receiver operator curve (AUROC) for the ANN $(92.6 \%, 95 \% \mathrm{Cl} 91.1-94.1)$ was significantly greater than that of the Cox regression model $(86.9 \%, 95 \% \mathrm{Cl} 85.7-88.2)$, $p=0.0136$. Both models achieved acceptable calibration with Brier scores of 0.13 and 0.11 for the Cox model and ANN, respectively.

Conclusion: We demonstrate an ANN which is non-inferior to a Cox regression model but with potential for further development such that it can learn as new data becomes available. Deep learning techniques are particularly suited to complex datasets with non-linear solutions, which make them appropriate for use in conditions with a paucity of prior knowledge. Accurate prognostic models for SARS-CoV-2 can provide benefits at the patient, departmental and organisational level.

Keywords: COVID-19, Coronavirus, Machine learning, Artificial intelligence, Prognostication

*Correspondence: I.moore@imperial.ac.uk

${ }^{\dagger}$ Ahmed Abdulaal and Aatish Patel contributed equally to this work

${ }^{1}$ Chelsea and Westminster NHS Foundation Trust, 369 Fulham Road,

London SW10 9NH, UK

Full list of author information is available at the end of the article

\section{Background}

Severe acute respiratory syndrome coronavirus 2 (SARS$\mathrm{CoV}-2$ ) has led to healthcare crises in several countries and remains disruptive in several others [1]. Accurately predicting patient outcomes would aid clinical staff in allocating limited healthcare resources and establishing appropriate ceilings of care, thereby mitigating the 
pressure on hospital departments. It would also allow service managers and policy makers to respond efficiently to possible future surges of SARS-CoV-2, the magnitudes of which may otherwise be difficult to predict [2].

There are a variety of methods which can be used to develop prognostic models, ranging from logistic regression and survival analysis to more complex machine learning algorithms and deep learning [3]. As a consequence of the emergent interest in deep learning, a number of techniques have been developed within this field with respect to the diagnosis, treatment and prognosis of the COVID-19 disease, including densely connected neural networks, recurrent networks and generative adversarial networks [4]. There is currently no consensus as to which of these techniques yields the most robust prognostic models [5], and whilst several models have been developed at a time when they are urgently required, there are a number of limitations which have impeded their use [6]. Several of the current models have been found to be highly susceptible to bias. For example, many demonstrate sampling bias as they excluded patients with no outcome at the end of the study period, leading to reported mortality rates of between 8 and 59\% [6-9]. Others attempt to predict outcomes based on cross-sectional data, suggesting the outcome prediction is based on data which is likely collected at a different time to that for which the model is intended [10]. One model attempts to predict outcomes from the last measurements available in healthcare records [8]. Other limitations include the use of subjective predictors [6], small patient numbers [11], and considering suspected and confirmed SARS-CoV-2 cases as one group [12].

We aimed to develop and compare two separate predictive models using regression analysis and an artificial neural network (ANN) using the Transparent Reporting of a multivariable prediction model for Individual Prognosis or Diagnosis (TRIPOD) guidelines [13]. The models aim to predict the risk of death during admission in patients with SARS-CoV-2. We then compare the two techniques to establish whether deep learning could supplant classical methods in the context of an evolving pandemic.

\section{Methods}

\section{Participants}

All admitted patients with a laboratory diagnosis of SARS-CoV-2 during March 1-April 24, 2020 (i.e. a high prevalence period) from a single west London hospital were identified. Patients were included if they were admitted to hospital and diagnosed with SARS-CoV-2 based on real-time reverse transcriptase polymerase chain reaction (RT-PCR, proprietary
Public Health England Assay until 10 March 2020, then AusDiagnostics $^{\circledR}$, Australia, assay thereafter). No patients were excluded.

Inpatients had their symptoms and clinical course documented in their electronic healthcare record (EHR) by the admitting clinical team (Millennium: Cerner Corporation, Kansas City, Missouri). Demographic and clinical data were extracted retrospectively from the EHRs for all patients included in the analysis by the infectious diseases team. Patient outcomes were followed up until death or discharge.

\section{Outcome measure}

Outcome was defined as death occurring during hospital admission for patients who were admitted with a laboratory confirmed diagnosis of SAR-CoV-2.

\section{Predictors}

Predictors were chosen in concordance with previously published literature [10, 14, 15], and included demographic details (age and sex), comorbidities (chronic respiratory disease, obesity, hypertension, diabetes, ischaemic heart disease, cardiac failure, chronic liver disease, chronic kidney disease, and history of a cerebrovascular event), symptomatology (fever, cough, dyspnoea, myalgia, abdominal pain, diarrhoea and vomiting, confusion, collapse, and olfactory change), and the number of days of symptoms prior to admission. Length of hospital stay to discharge, or death, was recorded for all patients to allow for survival analysis in the Cox regression model. Smoking history and ethnicity data were not included in the predictive models due to $28.9 \%$ and $23.4 \%$ of patients having missing data for these fields, respectively.

Age and number of days of symptoms prior to admission were continuous variables. All other predictors were encoded as binary presence features. Sex was converted to a binary feature where 0 and 1 represented male and female patients, respectively. Predictors were chosen such that they can be elicited on first contact with a healthcare worker. The intended use, for both models, is therefore an outcome prediction based on clinical admission data.

\section{Statistical analysis}

Patient baseline characteristics were described by mean and median for continuous variables and frequency and proportion for categorical variables. Log rank analysis was applied to the whole dataset to report unadjusted associations between each predictor and the outcome. Age was not normally distributed and was normalised by calculating its fractional ranks and then using an inverse density function. We then used an independent samples t-test to compare age by outcomes. Number of 
days of symptoms prior to hospital admission (NOD) were also not normally distributed and a Mann-Whitney $U$ test was carried out to compare NOD between outcome groups. Multivariable Cox regression analysis was then applied to contextualise the predictors in relation to each other.

\section{Cox regression predictive model}

To create a predictive model for death in SARS-CoV-2, we randomly split the dataset into training $(80 \%)$ and test $(20 \%)$ sets. As others have demonstrated, the optimal proportion of the dataset partitioned for training depends on the full dataset size and classification accuracy, with higher accuracies and smaller dataset sizes requiring a larger majority of the data for training the model [16]. However, a range of proportions for the training set were trialled during the training phase of model development for both the Cox regression and ANN models. The training/test set portions yielding the highest average area under the receiver operator curve (AUROC) during training cross-validation were used in the testing phase, and their results are reported in this analysis. On the training set, we used a parsimonious model building approach using the clinically relevant demographic, comorbidity and symptomatology features identified. All predictors were included in a Cox regression model irrespective of whether they were significant in univariable log-rank analysis. Using k-fold cross-validation on the training set, we chose the model with the lowest Akaike information criterion (AIC) score and highest concordance index (c-index) [17]. Subsequently, predictors which were not significantly associated with death were removed using backwards elimination. This generated a list of predictors making up a predictive model. We then assessed the performance of the model by calculating the survival function at the third quartile of length of stay for patients in the test set, as length of stay was not normally distributed. Since predicting mortality is a binary classification problem, a standard threshold of 0.5 (50\%) was used to predict mortality. For example, if the model predicts a patient-specific mortality of $60 \%$, this is interpreted as a "positive prediction", in that the patient is likely to die. Accuracy, sensitivity, specificity, positive predictive value, and negative predictive value were computed. Using $\mathrm{k}$-fold cross-validation on the whole dataset allowed for a calculation of a mean c-index with 95\% confidence intervals (CI). Model calibration was assessed graphically using a calibration curve and numerically with a Brier score, which represents the mean squared error for a probabilistic forecast, with a lower score representing more calibrated predictions [18].

\section{Artificial neural network predictive model}

The dataset was again randomly split into training $(80 \%)$ and test $(20 \%)$ sets. To maximise network learning efficiency, feature-wise normalisation was used. Each feature in the input data was centred around 0 by subtracting the mean of the feature, and then dividing it by its standard deviation [19]. The open-source TensorFlow machine learning library [20] was used to construct the ANN. To optimise the model, we adjusted hyperparameters (the number and size of layers, batch-size, dropout, and regularisation) using $\mathrm{k}$-fold cross validation on the training set. The ANN was designed to achieve maximal performance on cross-validation. Once the model architecture was established, we retrained the ANN on the entire training set, before finally validating its performance on the test set. We calculated the same performance metrics and assessed calibration in the same manner as the Cox regression model. The performance profiles of the models were then compared, and an efficient implementation for Delong's algorithm (which is an algorithm used to compare the area under two or more correlated receiver operator curves) was used to compare the AUROC between both models [21, 22]. Figure 1 illustrates a summary of the model development and assessment methodology.

\section{Results \\ Participants}

398 patients were identified, of which 95 died during hospital admission with SARS-CoV-2. 3/398 (0.75\%) were still inpatients at the end of the follow-up period. There were no missing data in the variables used for analysis. Table 1 summarises the demographic, comorbidity, and symptomatology characteristics of the cohort, with the log rank (Mantel-Cox) analysis of all predictors. The mean age was 63.2 years and there was a statistically significant relationship between age and death $(p<0.001)$. The median number of days of symptoms prior to admission was 7 days (IQR 2-10). There was no significant association between number of days of symptoms and death $(p=0.09)$.

\section{Cox regression model Development}

Table 2 shows the association of all predictors with survival following multivariable analysis. Following backwards elimination on the training set $(318 / 398)$, the remaining variables of significance were: age, sex, obesity, ischaemic heart disease, cardiac failure, chronic liver disease, chronic kidney disease, cerebrovascular event history, cough, dyspnoea, abdominal pain, confusion 


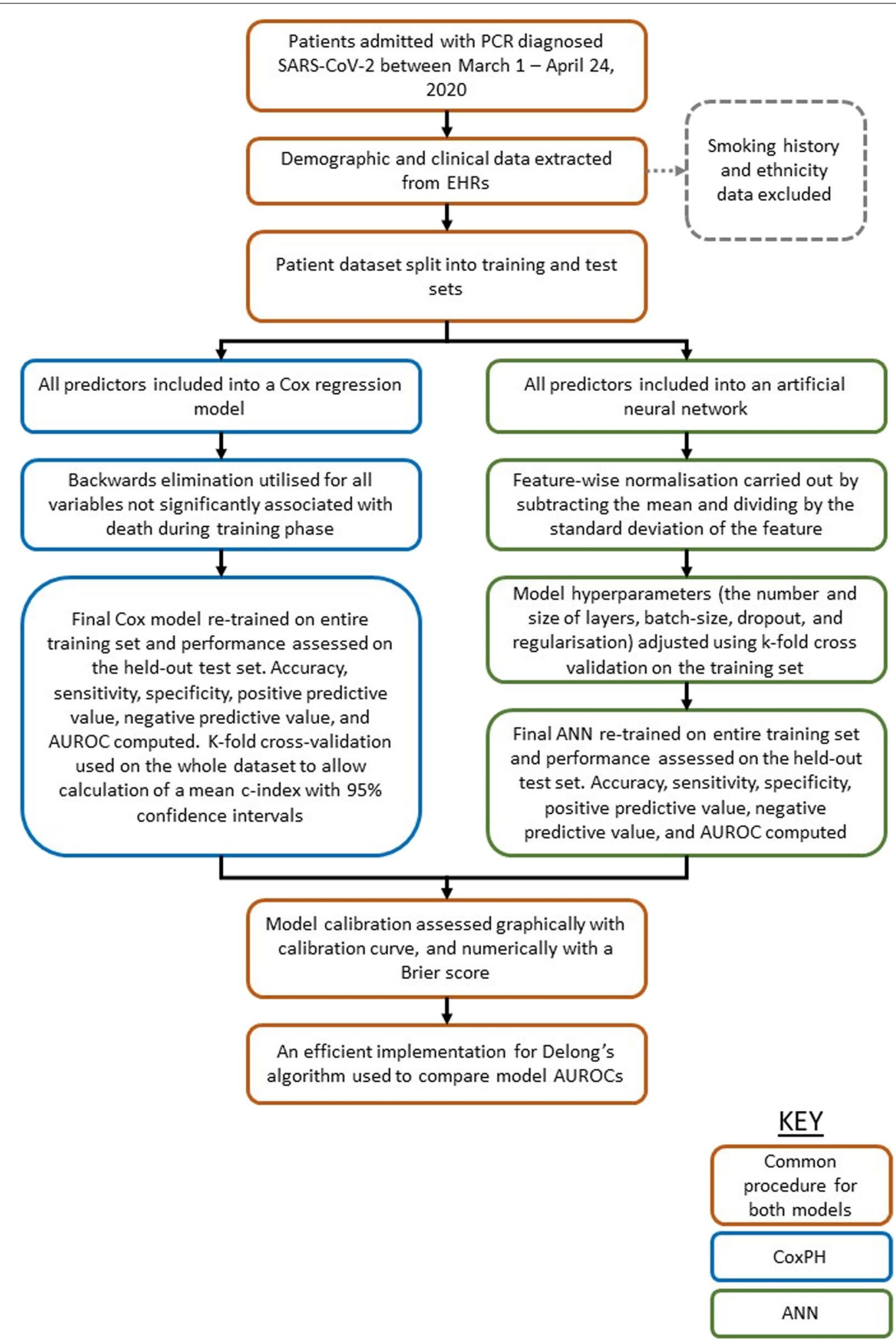

Fig. 1 Summary of the methodology used in the development and assessment of two prognostic models for patients admitted with SARS-CoV-2 in a West London population, during March 1-April 23, 2020. ANN artificial neural network, CoxPH Cox regression model 
Table 1 Summary of demographics, comorbidities, symptoms, and outcomes of 398 patients admitted with SARS-CoV-2 in a West London population, during March 1-April 23, 2020

\begin{tabular}{|c|c|c|c|}
\hline & Number (\%) & Chi-Square & $p$ value \\
\hline \multicolumn{4}{|l|}{ Demographics } \\
\hline Age (mean) & 63.2 years & & \\
\hline $\operatorname{Sex}(M)$ & $223(56.0 \%)$ & 3.54 & \\
\hline \multicolumn{4}{|l|}{ Comorbidities } \\
\hline Cardiac failure & $22(5.5 \%)$ & 79.56 & $<0.005$ \\
\hline Cerebrovascular event & $29(7.3 \%)$ & 24.14 & $<0.005$ \\
\hline Chronic kidney disease & $33(8.3 \%)$ & 49.25 & $<0.005$ \\
\hline Chronic liver disease & $6(1.5 \%)$ & 5.915 & 0.015 \\
\hline Chronic lung disease & $84(21.1 \%)$ & 6.01 & 0.01 \\
\hline Diabetes & $104(26.1 \%)$ & 4.39 & 0.036 \\
\hline Hypertension & $147(36.9 \%)$ & 17.88 & $<0.005$ \\
\hline Ischaemic heart disease & $47(11.8 \%)$ & 37.63 & $<0.005$ \\
\hline Obesity & $15(3.8 \%)$ & 0.015 & 0.903 \\
\hline \multicolumn{4}{|l|}{ Symptoms } \\
\hline Abdominal pain & $40(10.1 \%)$ & 2.6 & 0.11 \\
\hline Collapse & $37(9.3 \%)$ & 55.5 & $<0.005$ \\
\hline Confusion & $59(14.8 \%)$ & 117.35 & $<0.005$ \\
\hline Cough & $24762.1 \%)$ & 3.05 & 0.081 \\
\hline Diarrhoea and vomiting & $105(26.4 \%)$ & 4.64 & 0.031 \\
\hline Dyspnoea & $223(56.0 \%)$ & 15.88 & $<0.005$ \\
\hline Fever & $216(54.3 \%)$ & 0.12 & 0.73 \\
\hline Myalgia & $68(17.1)$ & 0.002 & 0.97 \\
\hline Olfactory change & $36(9.0 \%)$ & 1.11 & 0.29 \\
\hline Length of stay (median) & 5 days & & \\
\hline $\begin{array}{l}\text { Number of days of symptoms } \\
\text { prior to admission (median) }\end{array}$ & 7 days & & \\
\hline \multicolumn{4}{|l|}{ Outcome } \\
\hline Death & $95(23.9 \%)$ & & \\
\hline
\end{tabular}

The association of each predictor with death following log rank analysis (reported with the Chi-square statistic) is shown

and collapse (Fig. 2). Goodness of fit testing showed an $\mathrm{AIC}=572.93$ with a c-index of 0.90 on the training set.

\section{Specification}

We generated a model that calculates the hazard function determined by the following significant variables and their coefficients:

$$
\begin{aligned}
h(t)= & h_{0}(t) \times \exp ((0.05 \times \text { age })+(-0.78 \times \mathrm{sex}) \\
& +(1.48 \times \text { obesity }) \\
& +(0.93 \times \text { ischaemicheartdisease }) \\
& +(0.91 \times \text { cardiacfailure }) \\
& +(1.36 \times \text { chronicliverdisease }) \\
& +(1.03 \times \text { chronickidneydisease }) \\
& +(0.78 \times \text { cerebrovascularevent }) \\
& +(1.01 \times \text { cough })+(1.54 \times \text { dypsnoea }) \\
& +(-1.45 \times \text { abdominalpain }) \\
& +(1.81 \times \text { confusion })+(1.35 \times \text { collapse }))
\end{aligned}
$$

Median length of stay was 7 days with the upper quartile being 13 days. Therefore, to predict outcomes, the survival function was calculated for patients at day 13 in the test set.

\section{Performance}

K-fold cross validated mean c-index on the training set was $89.0 \%$ (95\% CI 84.2-94.3). When applied to the test set, the Cox regression model provided a sensitivity of $50.0 \%$ (95\% CI $28.2-71.8$ ), specificity of $96.6 \%$ (95\% CI $88.1-99.6)$, positive predictive value of $84.6 \%$ (95\% CI 57.0-95.8) and negative predictive value of $83.6 \%$ (95\% CI 77.0-88.6) with an accuracy of $83.75 \%$ (95\% CI 73.8-91.1). The c-index was $86.9 \%$ (95\% CI 85.7-88.2). The final model had a Brier score of 0.13 .

\section{ANN model \\ Development}

We applied the ANN to the training set and adjusted the hyperparameters (layers, neurones, drop out, batch size, regularisation and epoch number) to achieve a model architecture providing the highest accuracy, AUROC and the lowest loss as measured by binary cross-entropy on the validation set. Once architecture was optimised, the model was retrained on the entire training set and evaluated on the test set. Figure $3 \mathrm{dem}$ onstrates the average AUROC by training proportion for both the Cox regression and ANN models during training cross-validation. 
Table 2 Multivariable Cox regression analysis in 398 patients admitted with SARS-CoV-2 in a West London population, during March 1-April 23, 2020

\begin{tabular}{|c|c|c|c|c|}
\hline Variable & Hazard ratio & Lower $95 \% \mathrm{Cl}$ & Upper $95 \% \mathrm{Cl}$ & $p$ value \\
\hline Age & 1.05 & 1.03 & 1.07 & $<0.005$ \\
\hline $\operatorname{Sex}(F)$ & 0.6 & 0.36 & 1 & 0.05 \\
\hline Cardiac failure & 2.92 & 1.52 & 5.62 & $<0.005$ \\
\hline Cerebrovascular event & 2.36 & 1.28 & 4.36 & 0.01 \\
\hline Chronic kidney disease & 2.32 & 1.31 & 4.1 & $<0.005$ \\
\hline Chronic liver disease & 3.52 & 1.2 & 10.35 & 0.02 \\
\hline Chronic respiratory disease & 0.9 & 0.53 & 1.53 & 0.7 \\
\hline Diabetes & 1.41 & 0.86 & 2.3 & 0.17 \\
\hline Hypertension & 1.02 & 0.64 & 1.63 & 0.93 \\
\hline Ischaemic heart disease & 2.09 & 1.29 & 3.4 & $<0.005$ \\
\hline Obesity & 2.74 & 0.97 & 7.7 & 0.06 \\
\hline NOD & 1.01 & 0.97 & 1.05 & 0.65 \\
\hline Abdominal pain & 0.29 & 0.08 & 1.02 & 0.05 \\
\hline Collapse & 4.21 & 2.4 & 7.41 & $<0.005$ \\
\hline Confusion & 6.03 & 3.5 & 10.41 & $<0.005$ \\
\hline Cough & 1.88 & 1.1 & 3.2 & 0.02 \\
\hline Diarrhoea/vomiting & 1.32 & 0.68 & 2.54 & 0.41 \\
\hline Dyspnoea & 3.49 & 1.93 & 6.32 & $<0.005$ \\
\hline Fever & 1.88 & 1.13 & 3.13 & 0.02 \\
\hline Myalgia & 1.43 & 0.69 & 2.94 & 0.33 \\
\hline Olfactory change & 0.87 & 0.31 & 2.47 & 0.79 \\
\hline
\end{tabular}

NOD Number of days of symptoms prior to hospital admission

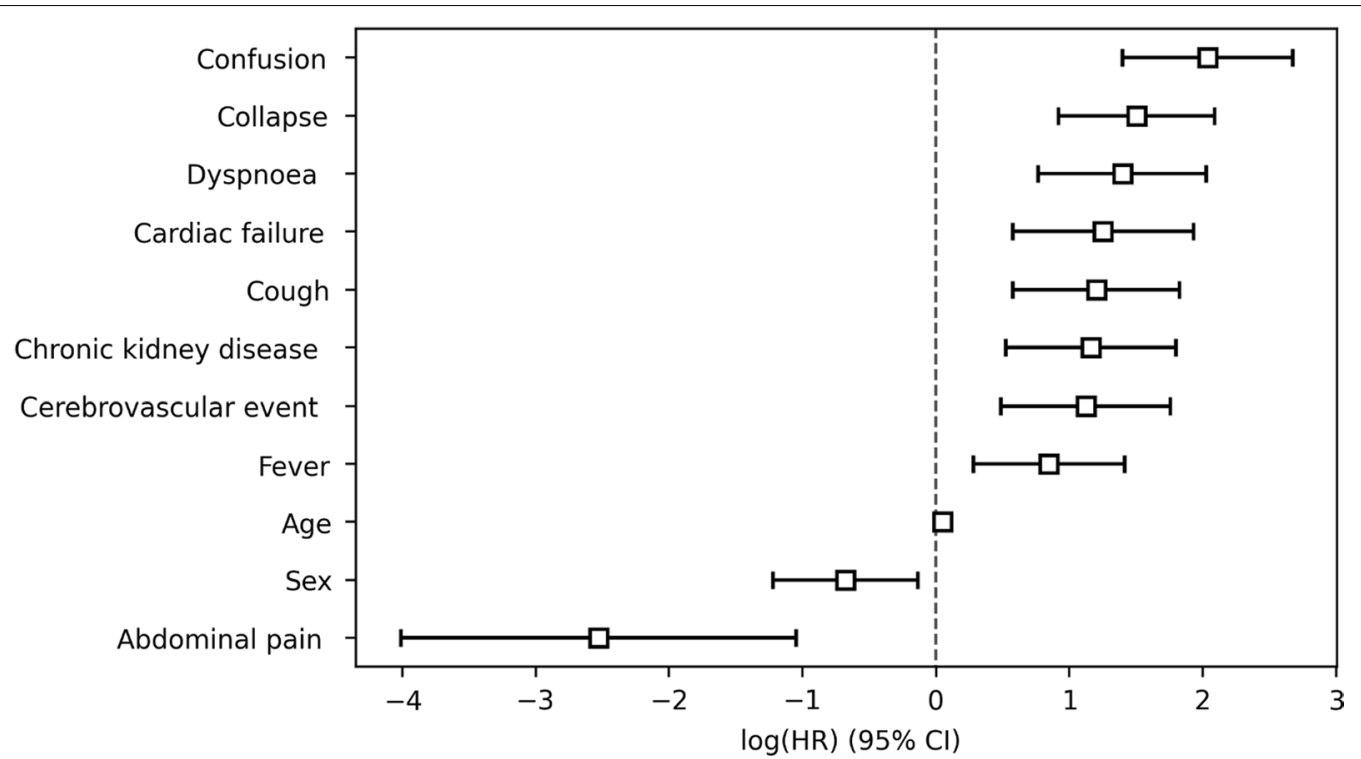

Fig. 2 Cox prognostic model of demographics, comorbidities and symptoms, and the log hazard ratio of death in patients admitted with SARS-CoV-2 in a West London population, during March 1-April 23, 2020 


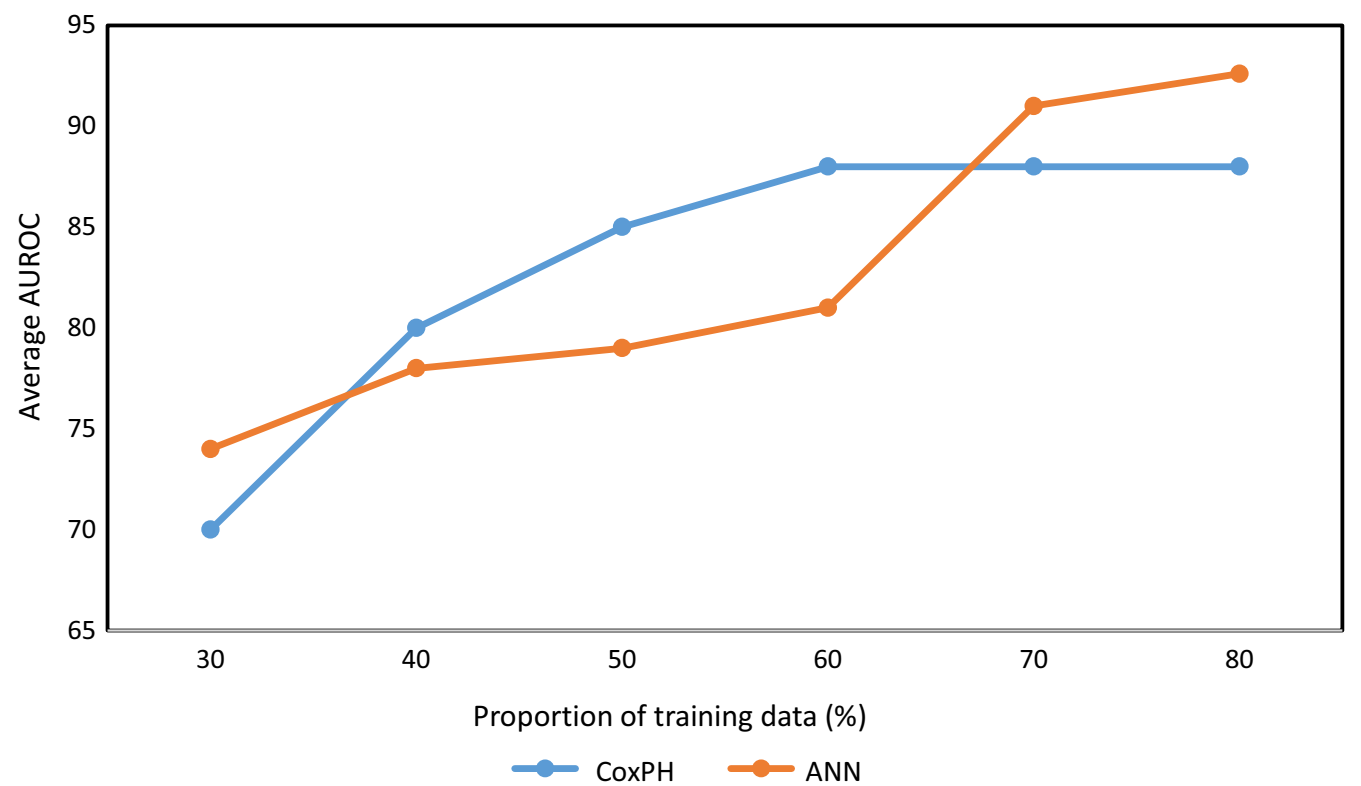

Fig. 3 The average area under the receiver operator curve (AUROC) achieved by a Cox regression model and an Artificial Neural Network for a range of training set proportions in patients admitted with SARS-CoV-2 in a West London population, during March 1-April 23, 2020. ANN artificial neural network, CoxPH Cox regression model

\section{Specification}

Optimal validation results were achieved with an ANN with 21 units in the input layer to match the input dimension of the dataset, that is, the total number of predictors. Two further hidden layers were used with 8 and 4 units, respectively. A dropout of $20 \%$ was applied to the input layer and both hidden layers to prevent overfitting. Regularisation did not substantively improve results and therefore no regularisation techniques were used. Figure 4 demonstrates the average impact on model output magnitude by each predictor.

\section{Performance}

The final ANN architecture provided an accuracy of $88.1 \%$ and AUROC of $90.9 \%$ on cross validation. When applied to the test set, the model provided a sensitivity of $64.7 \%$ (95\% CI 38.3-85.8), specificity of $96.8 \%$ (95\% CI 89.0-99.6), positive predictive value of $84.6 \%$ (95\% CI 57.4-95.7) and negative predictive value of $91.4 \%$ (95\% CI 84.2-95.1), with an accuracy of 90.0\% (95\% CI 81.2-95.6). The AUROC on the test set was $92.6 \%$ (95\% CI 91.1-94.1). The ANN had a Brier score of 0.11. Table 3 shows the performance metrics of each model. Figure 5 demonstrates the calibration of each model.

\section{Discussion}

Two models were developed in accordance with TRIPOD methodology to predict death during hospital admission among SARS-CoV-2 patients. Both models demonstrate acceptable sensitivity and good specificity. Although both have good accuracy, the ANN has significantly greater discriminatory ability. Both models demonstrate acceptable calibration. Developing robust prognostic models for SARS-CoV-2 has benefits for the patient, medical departments, and hospital organisations.

Previous literature reports mixed performance of machine learning and deep learning techniques when compared to regression analysis [5]. Whilst machine learning does not obviate the need for classical methods [23], machine learning techniques have been shown to perform significantly better than classical regression models in high-dimensionality datasets [24]. Furthermore, ANNs have been shown to perform well on datasets of varying size [25-27]. Our results support the use of an ANN in a moderate sized, high-dimensional dataset, whilst having a non-inferior performance profile to a Cox regression model.

The Cox regression model used 11 predictors to calculate survival function, whilst the ANN uses all 21 input features, and attributes different weightings to each feature. Both models identify confusion, collapse, dyspnoea, cough, chronic kidney disease, heart failure, cerebrovascular event history, fever, and sex as more significant 


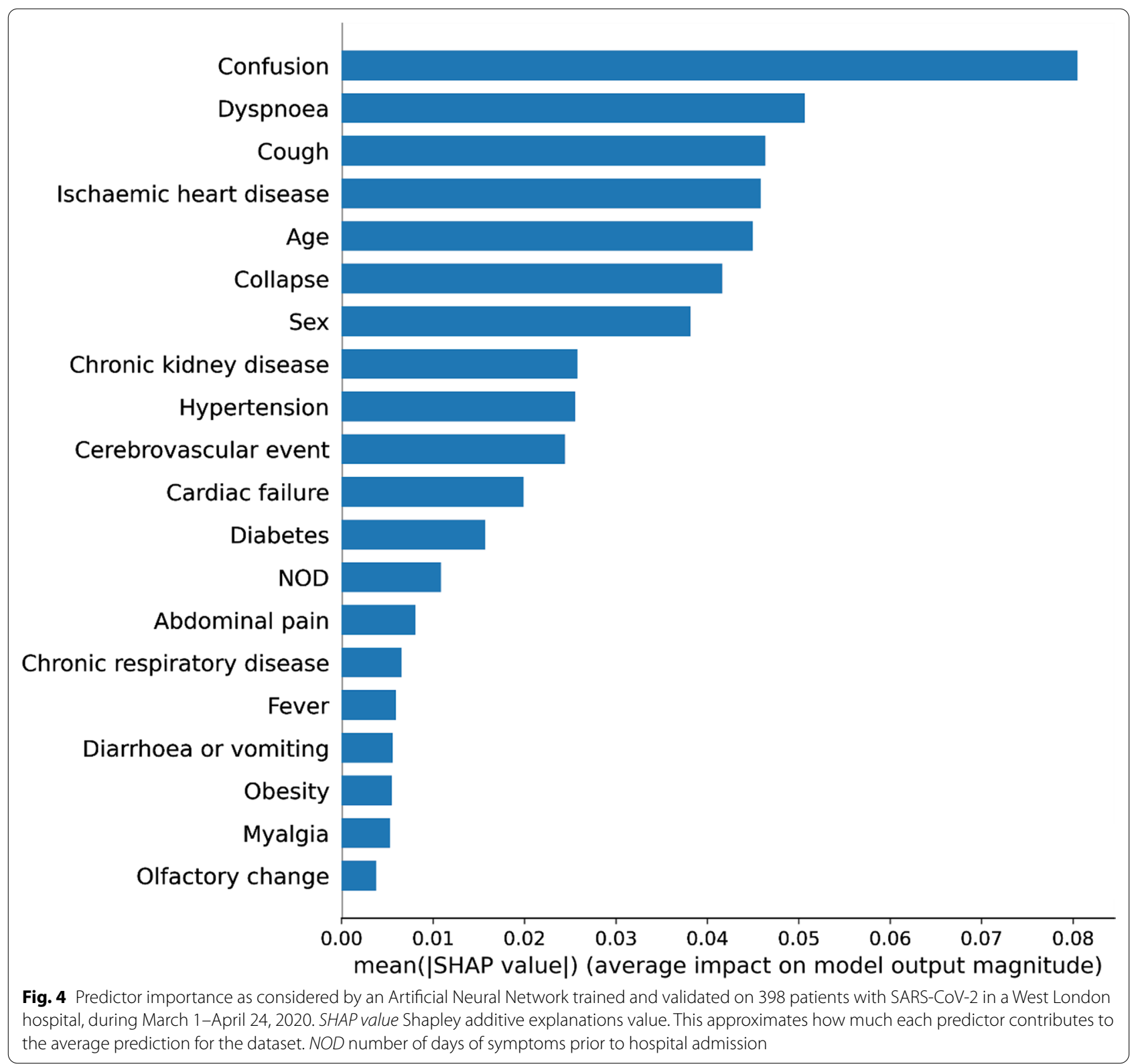

Table 3 Performance of the Cox regression model and an ANN on 398 patients with SARS-CoV-2 in a West London hospital, during March 1-April 24, 2020

\begin{tabular}{lclc}
\hline & Cox regression model (95\% Cl) & ANN model (95\% Cl) & $\begin{array}{l}\text { Cox } \\
\text { regression } \\
\text { vs ANN } \\
\text { model }\end{array}$ \\
\hline Sensitivity & & \\
Specificity & $50.0 \%(28.2-71.8)$ & $64.7 \%(38.3-85.8)$ \\
Positive predictive value & $96.6 \%(88.1-99.6)$ & $96.8 \%(89-99.6)$ & $84.6 \%(57.4-95.7)$ \\
Negative predictive value & $84.6 \%(57.0-95.8)$ & $91.4 \%(84.2-95.1)$ & $90.0 \%(81.2-95.6)$ \\
Accuracy & $83.6 \%(77.0-88.6)$ & $92.6 \%(91.1-94.1)$ & $Z=12.021$, \\
AUROC & $83.75 \%(73.8-91.1)$ & & $P<0.001$ \\
& $86.9 \%(85.7-88.2)$ & &
\end{tabular}

$A N N$ artificial neural network, $A U R O C$ area under the receiver operating characteristic curve 


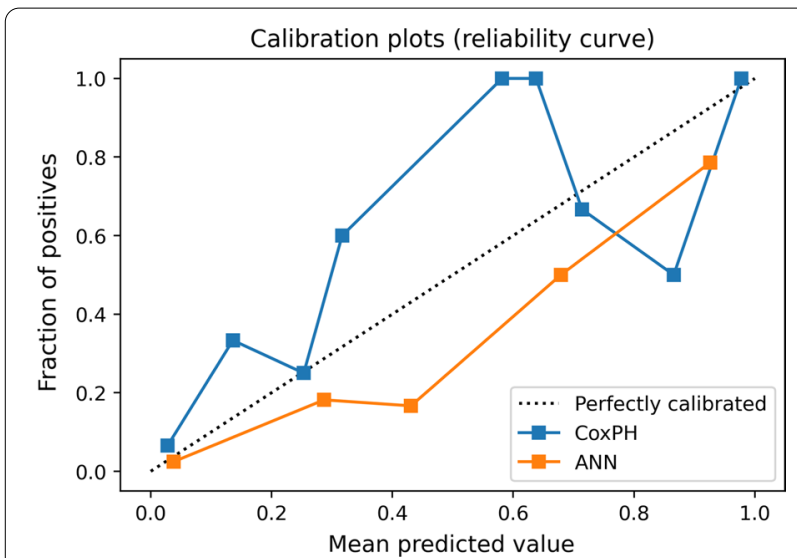

Fig. 5 Calibration of a Cox regression model and ANN on 398 patients with SARS-CoV-2 in a West London hospital, during March 1-April 24, 2020. ANN artificial neural network, COXPH Cox regression model

predictors of mortality. The ANN additionally identifies ischaemic heart disease and hypertension as important features. Abdominal pain is considered to have little effect on model output by the ANN, which is a significant 'protective factor' in the Cox model. In this context, abdominal pain may represent a milder form of SARS$\mathrm{CoV}-2$. These variables, in particular the comorbidities, have been shown to be associated with mortality in the current literature, such as the ISARIC protocol, which analysed 20,133 SARS-CoV-2 positive patients [28].

The Cox regression model accounts for censored patients in the study and therefore no patients were excluded on account of not having a recorded outcome at the end of the follow-up period. This avoids the introduction of sampling bias. The predictors chosen for inclusion in both models can be accrued from an initial encounter with a healthcare worker and relate to the underlying clinical condition of each patient. This has a dual benefit. Firstly, this standardises the data-collection process and ensures both models are compared on a congruent dataset. Secondly, the nature of the predictors means that the intended use of the models is clear in that they both produce a point-of-admission mortality prediction, which is particularly applicable to the development of medical calculators. The models analyse the outcomes for laboratory confirmed SARS-CoV-2 patients, eliminating potential bias introduced by including suspected cases who are subsequently diagnosed with other conditions.

The predictive models here do have several limitations, however. There are a variety of haematological and radiological predictors which have been associated with SARS-CoV-2 outcomes which are not included in our models $[29,30]$. Whilst our current models can produce point-of-admission outcome predictions due to the relative ease of collecting demographic, comorbidity and symptom data, additional clinical parameters could be introduced in future to improve the predictive accuracy of the models. We could not account for patients who were admitted for, and diagnosed with SARS-CoV-2, but may have died due to another comorbidity. However, this likely represents a minority of patient deaths. The Cox regression model predicts survival function at day 13; whilst this accounts for the majority of hospital admission lengths, predicting survival in this way may overestimate survival chance for outliers who died at a later date. In contrast, the ANN model produces an overall risk prediction irrespective of length of admission. However, given the median length of stay of 7 days with an upper quartile of 13 days, predictions from the ANN should be used cautiously for longer lengths of hospital stay. There may be a delay between patient presentation and obtaining a laboratory diagnosis. Therefore, whilst it is possible to use either model at the point of admission, the prediction should only be applied to patients who have a confirmed diagnosis of SARS-CoV-2. Finally, data was collected at a single site during a period of high prevalence, and therefore results should be generalised with caution to other populations and those with a different SARS-CoV-2 prevalence.

A prospective, multi-centre analysis is required to further validate the model and improve generalisability of results. Machine learning techniques are ideal for fluctuating environments as they can adapt to new data. For example, using online/active learning, an ANN can train incrementally by being fed data instances sequentially. Each step is relatively fast and cheap, meaning the system can continuously learn as more data is available. This represents a major advantage relative to static statistical models [31]. Future research should focus on implementing adaptive workflows to allow for multi-site data collection, cross-population train/test modelling, and flexible systems which learn incrementally. Additionally, multimodal data (such as encoded radiographic data), and other potentially important parameters such as hospital capacity, testing capacity/rate and income versus commodities (poverty) can all be incorporated to produce more generalisable, highly-performant models [32, 33]. Furthermore, deep learning techniques such as recurrent neural networks can be used for time-series analysis, and therefore account for important events such as ICU admission as they occur. This may represent an additional avenue for further research. Finally, other decision points in SARS-CoV-2 patient journeys need to be predicted, and adapting the models to predict need for antibacterial agents for secondary infection [34], or for steroids where indicated [35], are clear avenues for exploration. 


\section{Conclusion}

Accurate prognostic models for SARS-CoV-2 can provide benefits at the patient, departmental and organisational level. Such models could optimise the response to possible future surges of SARS-CoV-2. We demonstrate an ANN which is non-inferior to a Cox regression model but has the potential for further development such that it can learn as new data becomes available. Deep learning techniques are particularly suited to complex datasets with non-linear solutions, which make them appropriate for use in conditions with a paucity of prior knowledge, such as in SARS-CoV-2 infection.

\begin{abstract}
Abbreviations
AIC: Akaike information criterion; ANN: Artificial neural network; AUROC: Area under receiver operator curve; $\mathrm{Cl}$ : Confidence interval; c-index: Concordance index; NOD: Number of days; SARS-CoV-2: Severe acute respiratory syndrome coronavirus 2; TRIPOD: The Transparent Reporting of a multivariable prediction model for Individual Prognosis or Diagnosis; UK: United Kingdom.
\end{abstract}

\section{Acknowledgements \\ EC is supported by Economic and Social Science Research Council (ESRC) and the National Institute for Health Research Health Protection Research Unit (HPRU) in Healthcare Associated Infections and Antimicrobial Resistance, UK Department of Health [HPRU-2012-10047] in partnership with Public Health England. LSPM acknowledges support from the National Institute of Health Research (NIHR) Imperial Biomedical Research Centre (BRC) and the National Institute for Health Research HPRU in Healthcare Associated Infection and Antimicrobial Resistance at Imperial College London in partnership with Public Health England. The views expressed in this publication are those of the authors and not necessarily those of the NHS, the National Institute for Health Research, or the UK Department of Health.}

\section{Authors' contributions}

Conceptualization: AA, AP, LSPM. Data curation: AA, AP, EC. Formal Analysis: AA, AP, LSPM. Methodology: AA, AP, LSPM. Validation: AA, AP, LSPM. Visualization: $A A, A P$. Writing - original draft: AA, AP. Writing - review and editing: AA, AP, EC, SD, SAA, GWD, NM, LSPM. All authors read and approved the final manuscript.

\section{Funding}

This research did not receive any grant from funding agencies in the public or commercial sectors.

\section{Availability of data and materials}

The datasets analysed during the current study and further details on gaining access to the intervention reported within this study are available from the first author (AA ahmed.abdulaal@nhs.net) on reasonable request, as long as this meets local ethics and research governance criteria. The open source machine learning framework Tensorflow 2.1.0 (https://github.com/tensorflow /tensorflow) was used to develop the neural network. The architecture was written in the python programming language (Python 3.7.7). Scikit-learn 0.22 and its dependencies were utilised to create the data pre-processing pipeline and to create the graphs in this analysis.

\section{Ethics approval and consent to participate}

Data was collected as part of routine care by the responsible clinical team. Data was anonymised at the point of extraction by the care team and no patient identifiable data is reported in this analysis. The study protocol was approved by the Antimicrobial Stewardship Group at Chelsea \& Westminster NHS Foundation Trust and this was confirmed (i) as a service development (Reference AP_89) and (ii) that individual written consent to participate was not required by the Research \& Development Office of Chelsea \& Westminster NHS Foundation Trust. The analysis was conducted in accordance with the Helsinki declaration.

\section{Consent for publication}

Not applicable.

\section{Competing interests}

All authors have completed the ICMJE form for uniform disclosure Form for Disclosure of Potential Conflicts of Interest and declare the following: EC has received speaker fees from bioMerieux (2019). NM has received speaker fees from Beyer (2016) and Pfizer (2019-2020) and received educational support from Eumedica (2016) and Baxter (2017). LSPM has consulted for DNAelectronics (2015-18), Dairy Crest (2017-2018), bioMerieux (2013-2020), Umovis Lab (2020), received speaker fees from Profile Pharma (2018-2019) and Pfizer (2018-2020), received research grants from the National Institute for Health Research (2013-2020), CW + Charity (2018-2020), and Leo Pharma (2016), and received educational support from Eumedica (2016-2018). AA and AP none to declare.

\section{Author details}

${ }^{1}$ Chelsea and Westminster NHS Foundation Trust, 369 Fulham Road, London SW10 9NH, UK. ${ }^{2}$ National Institute for Health Research Health Protection Research Unit in Healthcare Associated Infections and Antimicrobial Resistance, Imperial College London, Hammersmith Campus, Du Cane Road, London W12 ONN, UK. ${ }^{3}$ King Faisal Specialist Hospital and Research Centre, Riyadh, Saudi Arabia. ${ }^{4}$ Johns Hopkins University, Baltimore, MD, USA. ${ }^{5}$ North West London Pathology, Imperial College Healthcare NHS Trust, Fulham Palace Road, London W6 8RF, UK.

Received: 6 August 2020 Accepted: 4 November 2020

Published online: 19 November 2020

\section{References}

1. Zhou F, Yu T, Du R, Fan G, Liu Y, Liu Z, et al. Clinical course and risk factors for mortality of adult inpatients with COVID-19 in Wuhan, China: a retrospective cohort study. Lancet. 2020;395(10229):1054-62.

2. Phua J, Weng L, Ling L, Egi M, Lim C-M, Divatia JV, et al. Intensive care management of coronavirus disease 2019 (COVID-19): challenges and recommendations. Lancet Respir Med. 2020;8(5):506-17.

3. Abdulaal A, Patel A, Charani E, Denny S, Mughal N, Moore L. Prognostic modeling of COVID-19 using artificial intelligence in the United Kingdom: model development and validation. J Med Internet Res. 2020;22(8):e20259. https://doi.org/10.2196/20259.

4. Jamshidi M, Lalbakhsh A, Talla J, Peroutka Z, Hadjilooei F, Lalbakhsh P, et al. Artificial intelligence and COVID-19: deep learning approaches for diagnosis and treatment. IEEE Access. 2020;8:109581-95.

5. Christodoulou E, Ma J, Collins GS, Steyerberg EW, Verbakel JY, Van Calster B. A systematic review shows no performance benefit of machine learning over logistic regression for clinical prediction models. J Clin Epidemiol. 2019;110:12-22. https://doi.org/10.1016/j.jclinepi.2019.02.004.

6. Wynants L, Van Calster B, Bonten MMJ, Collins GS, Debray TPA, De Vos M, et al. Prediction models for diagnosis and prognosis of covid-19 infection: systematic review and critical appraisal. BMJ. 2020;369:m1328.

7. Xie J, Hungerford D, Chen H, Abrams ST, Li S, Wang G, et al. Development and external validation of a prognostic multivariable model on admission for hospitalized patients with COVID-19. medRxiv. Cold Spring Harbor Laboratory Press; 2020;2020.03.28.20045997.

8. Yan L, Zhang H-T, Xiao Y, Wang M, Sun C, Liang J, et al. Prediction of criticality in patients with severe Covid-19 infection using three clinical features: a machine learning-based prognostic model with clinical data in Wuhan. medRxiv. Cold Spring Harbor Laboratory Press. 2020. https:// medrxiv.org/content/early/2020/03/03/2020.02.27.20028027.abstract.

9. Yuan M, Yin W, Tao Z, Tan W, Hu Y. Association of radiologic findings with mortality of patients infected with 2019 novel coronavirus in Wuhan, China. PLoS ONE. 2020;15(3):e0230548.

10. Shi $Y$, Yu $X$, Zhao $H$, Wang $H$, Zhao R, Sheng J. Host susceptibility to severe COVID-19 and establishment of a host risk score: findings of 487 cases outside Wuhan. Crit Care. 2020;24(1):108. https://doi.org/10.1186/s1305 4-020-2833-7

11. Jiang $X$, Coffee M, Bari A, Wang J, Jiang X, Huang J, et al. Towards an artificial intelligence framework for data-driven prediction of Coronavirus 
Clinical Severity. Comput Mater Contin Comput Mater Continua. 2020;62(3):537-51.

12. Lu J, Hu S, Fan R, Liu Z, Yin X, Wang Q, et al. ACP risk grade: a simple mortality index for patients with confirmed or suspected severe acute respiratory syndrome coronavirus 2 disease (COVID-19) during the early stage of outbreak in Wuhan, China. medRxiv. Cold Spring Harbor Laboratory Press; 2020;2020.02.20.20025510.

13. Collins GS, Reitsma JB, Altman DG, Moons KGM. Transparent reporting of a multivariable prediction model for individual prognosis or diagnosis (TRIPOD): the TRIPOD statement. Ann Intern Med. 2015;162(1):55-63.

14. Caramelo F, Ferreira N, Oliveiros B. Estimation of risk factors for COVID-19 mortality_preliminary results. medRxiv. 2020. https://doi. org/10.1101/2020.02.24.20027268v1.

15. Patel A, Charani E, Ariyanayagam D, Abdulaal A, Denny SJ, Mughal N, et al. New-onset anosmia and ageusia in adult patients diagnosed with SARSCoV-2 infection. Clin Microbiol Infect. 2020;26(9):1236-41.

16. Dobbin KK, Simon RM. Optimally splitting cases for training and testing high dimensional classifiers. BMC Med Genomics. 2011;4(1):31. https:// doi.org/10.1186/1755-8794-4-31.

17. Menni C, Valdes AM, Freidin MB, Sudre $C H$, Nguyen $L H$, Drew DA, et al. Real-time tracking of self-reported symptoms to predict potential COVID-19. Nat Med. 2020;1-4. https://www.nature.com/articles/s4159 1-020-0916-2

18. sklearn.metrics.brier_score_loss - scikit-learn 0.23.2 documentation. https://scikit-learn.org/stable/modules/generated/sklearn.metrics.brier _score_loss.html.

19. Liao Z, Carneiro G. On the importance of normalisation layers in deep learning with piecewise linear activation units. In: 2016 IEEE Winter Conference on Applications of Computer Vision, WACV 2016. Institute of Electrical and Electronics Engineers Inc:; 2016.

20. TensorFlow. https://www.tensorflow.org/.

21. Delong ER, Delong DM, Clarke-Pearson DL. Comparing the areas under two or more correlated receiver operating characteristic curves: a nonparametric approach. Biometrics. 1988;44:837.

22. Sun $X, X u$ W. Fast implementation of DeLong's algorithm for comparing the areas under correlated receiver operating characteristic curves. IEEE Signal Process Lett. 2014;21(11):1389-93.

23. Sargent DJ. Comparison of artificial neural networks with other statistical approaches. Cancer. 2001;91(S8):1636-42.

24. Feng J, Wang Y, Peng J, Sun M, Zeng J, Jiang H. Comparison between logistic regression and machine learning algorithms on survival prediction of traumatic brain injuries. J Crit Care. 2019;54:110-6.

25. Panesar SS, D'Souza RN, Yeh FC, Fernandez-Miranda JC. Machine learning versus logistic regression methods for 2-year mortality prognostication in a small, heterogeneous glioma database. World Neurosurg X. 2019:2:100012.
26. Bassi P, Sacco E, De Marco V, Aragona M, Volpe A. Prognostic accuracy of an artificial neural network in patients undergoing radical cystectomy for bladder cancer: a comparison with logistic regression analysis. BJU Int. 2007;99(5):1007-12. https://doi.org/10.1111/j.1464-410X.2007.06755.X.

27. Tomašev N, Glorot X, Rae JW, Zielinski M, Askham H, Saraiva A, et al. A clinically applicable approach to continuous prediction of future acute kidney injury. Nature. 2019;572(7767):116-9.

28. Docherty AB, Harrison EM, Green CA, Hardwick HE, Pius R, Norman L, et al. Features of 20133 UK patients in hospital with covid-19 using the ISARIC WHO Clinical Characterisation Protocol: prospective observational cohort study. BMJ. 2020;369:m1985. https://doi.org/10.1136/bmj.m1985.

29. Gong J, Ou J, Qiu X, Jie Y, Chen Y, Yuan L, et al. A tool to early predict severe 2019-Novel Coronavirus Pneumonia (COVID-19): a multicenter study using the risk nomogram in Wuhan and Guangdong. China Clin Infect Dis. 2020. https://doi.org/10.1093/cid/ciaa443.

30. Huang H, Cai S, LiY, Li Y, Fan Y, Li L, et al. Prognostic factors for COVID19 pneumonia progression to severe symptom based on the earlier clinical features: a retrospective analysis. medRxiv. Cold Spring Harbor Laboratory Press; 2020. https://medrxiv.org/content/early /2020/03/30/2020.03.28.20045989.abstract.

31. Hands-On Machine Learning with Scikit-Learn, Keras, and TensorFlow, 2nd edn. https://www.oreilly.com/library/view/hands-on-machine-learn ing/9781492032632/.

32. Santosh KC. Al-driven tools for coronavirus outbreak: need of active learning and cross-population train/test models on multitudinal/multimodal data. J Med Syst. 2020a;44(5):1-5. https://doi.org/10.1007/s10916-02001562-1.

33. Santosh KC. COVID-19 prediction models and unexploited data. J Med Syst. 2020b;44(9):1-4. https://doi.org/10.1007/s10916-020-01645-z.

34. Hughes S, Troise O, Donaldson H, Mughal N, Moore LS. Bacterial and fungal coinfection among hospitalised patients with COVID-19: a retrospective cohort study in a UK secondary care setting. Clin Microbiol Infect. 2020;26(10):1395-9.

35. Horby P, Lim WS, Emberson J, Mafham M, Bell J, Linsell L, et al. Effect of dexamethasone in hospitalized patients with COVID-19: preliminary report. N Engl J Med. 2020. https://doi.org/10.1056/NEJMoa2021436.

\section{Publisher's Note}

Springer Nature remains neutral with regard to jurisdictional claims in published maps and institutional affiliations.
Ready to submit your research? Choose BMC and benefit from:

- fast, convenient online submission

- thorough peer review by experienced researchers in your field

- rapid publication on acceptance

- support for research data, including large and complex data types

- gold Open Access which fosters wider collaboration and increased citations

- maximum visibility for your research: over $100 \mathrm{M}$ website views per year

At BMC, research is always in progress.

Learn more biomedcentral.com/submissions 